In Group 1, regardless of ethnicity, 29 (90.6\%) patients developed LN within 5 years or less from the onset of SLE symptoms, while the remaining $3(9.4 \%)$ developed LN after 5 years. In contrast, in Group 2, 24 (55.8\%) patients developed LN within 5 years or less while $19(44.2 \%)$ developed LN after 5 years. (P value $=0.002$

Further stratification was based on ethnicity and antibody $(A B)$ status to investigate the time to develop LN from SLE symptom onset: African ancestry with positive $A B$, African with negative $A B$, Asian with positive $A B$, Caucasian with positive $A B$ and Asian \& Caucasian with negative Ab. Analysis showed that of 29 (38.7\%) African ethnicity patients with the autoantibody combination, $19(65.5 \%)$, developed LN within 5 years. In comparison, 46 (61.3\%) patients, independent of ethnicity and $A B$ status, developed $L N$ after 5 years $(P$ value $=0.01)$

Conclusion: Patients with the unusual autoantibody combination of Sm, Ro \& RNP developed LN significantly earlier than patients who did not have this combination. This autoantibody combination was significantly over represented in the African ancestry patients. Our data suggests that African ancestry patients with this autoantibody combination are at increased risk of developing LN soon after SLE symptom onset and merit close monitoring for the development of renal disease. References:

[1] McCarty GA, Harley JB, Reichlin M. A distinctive autoantibody profile in Black female patients with lupus nephritis. Arthritis \& Rheumatism. 1993; 36 : 1560-1565

Table 1.1 Ethnicity with Autoantibody status showing the rate of progression into Lupus Nephritis.

\begin{tabular}{lcccc}
\hline & & \multicolumn{2}{c}{ Duration of LN onset } & Total \\
\cline { 3 - 4 } & & $\begin{array}{c}\text { Less than 5 years } \\
\text { after SLE onset }\end{array}$ & $\begin{array}{c}\text { More than 5 } \\
\text { years after SLE } \\
\text { onset }\end{array}$ & \\
\hline Ethnicity with & African with positive & 19 & 3 & 22 \\
AB status & African with negative & 9 & 4 & 13 \\
& Asian with positive & 5 & 0 & 5 \\
Total & Caucasian with positive & 5 & 0 & 5 \\
\hline & Other negatives & 15 & 15 & 30 \\
\hline
\end{tabular}

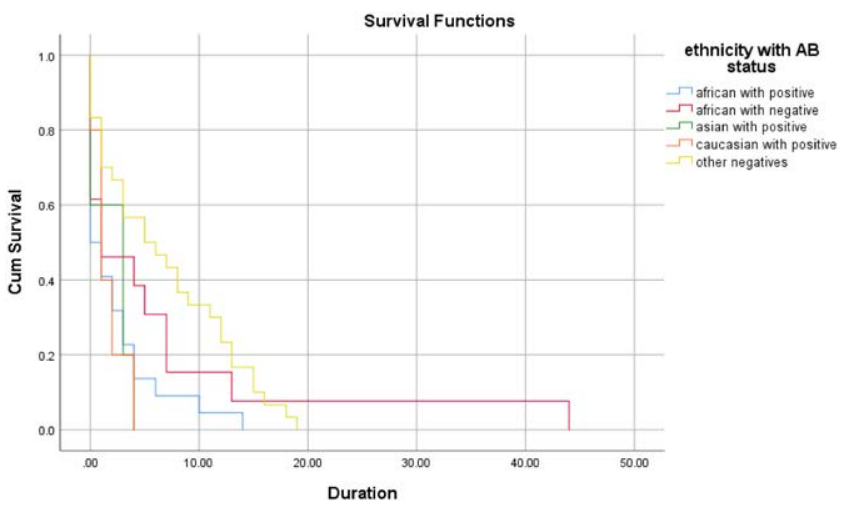

Graph 1. Ethnicity with Autoantibody status showing the rate of progression into Lupus Nephritis $(P$ value $=0.01)$

Disclosure of Interests: Majed Albirdisi: None declared, David d'cruz Grant/ research support from: GlaxoSmithKline, Shirish Sangle: None declared, Natasha Jordan: None declared

DOI: 10.1136/annrheumdis-2020-eular.4414

\section{THU0256 \\ CARDIAC INVOLVEMENT IN NEWLY DIAGNOSED SPANISH PATIENTS WITH SYSTEMIC LUPUS ERYTHEMATOSUS: DATA FROM THE RELES COHORT}

J. Álvarez Troncoso ${ }^{1}$, Á. Robles Marhuenda ${ }^{1}$, F. Mitjavila Villero ${ }^{2}$, F. J. García Hernández ${ }^{3}$, A. Marín Ballvé ${ }^{4}$, A. Castro ${ }^{5}$, G. Salvador Cervelló ${ }^{6}$, E. Fonseca ${ }^{7}$, I. Perales Fraile ${ }^{8}$, G. Ruiz-Irastorza ${ }^{9}$ on behalf of GRUPO RELES Investigators, Autoimmune Diseases Study Group (GEAS). ${ }^{1}$ Hospital Universitario La Paz, Department of Internal Medicine, Madrid, Spain; ${ }^{2}$ Hospital Universitari de Bellvitge, Department of Internal Medicine, L'Hospitalet de Llobregat, Barcelona, Spain; ${ }^{3}$ Hospital Virgen del Rocío, Department of Internal Medicine, Sevilla, Spain; ${ }^{4}$ Hospital Clínico Universitario Lozano Blesa IIIS Aragón. Grupo Autoinmunidad Clínica., Department of Internal Medicine, Zaragoza, Spain; ${ }^{5}$ Hospital Universitario Sant Joan de Reus, Department of Internal Medicine,
Tarragona, Spain; ${ }^{6}$ Hospital Universitario La Fe, Department of Internal Medicine, Valencia, Spain; ${ }^{7}$ Hospital de Cabueñes, Department of Internal Medicine, Gijón, Spain; ${ }^{8}$ Hospital Infanta Sofía, Department of Internal Medicine, Madrid, Spain; ${ }^{9}$ Hospital Universitario de Cruces, Unit of Autoimmune Diseases, Department of Internal Medicine, Baracaldo, Vizcaya, Spain

Background: Cardiac involvement is one of the most important causes of disability and mortality in patients with systemic lupus erythematosus (SLE). Transthoracic echocardiography (TTE) is a sensitive and specific technique in detecting cardiac abnormalities, particularly mild pericarditis, valvular lesions and myocardial dysfunction in SLE.

Objectives: Using data of patients from the inception cohort Registro Españo de Lupus Eritematoso Sistémico (RELES), we aimed to analyse the echocardiographic features of cardiac involvement of systemic lupus erythematosus (SLE). Methods: Prospective observational study on a multicenter Spanish inception cohort. Patients with SLE, diagnosed by the American College of Rheumatology (ACR) criteria, since January 2009, who had at least one TTE performed were selected. Demographic data, diagnostic criteria, follow-ups, treatments and SLEDAl were analyzed.

Results: We included 289 patients diagnosed with SLE with TTE performed The mean age was $40.5 \pm 1.9$ years, of which $86.9 \%$ (251) were women and $82.4 \%$ (238) Caucasian. The ACR score at diagnosis was $4.98 \pm 0.1$. Most frequent SLE manifestations were arthritis $(59.2 \%)$, photosensitivity $(49.5 \%)$, malar rash $(39.1 \%)$ and serositis $(31.1 \%)$. The main immunological findings were: ANA (97.6\%), anti-DNA (66.4\%), hypocomplementemia (58.7\%), antiphospholipid antibodies (31.5\%). One third (31.5\%) of the TTE performed were pathological. O these, $13.8 \%$ had pericardial effusion, $13.3 \%$ valvulopathy, $6.5 \%$ myocardial dysfunction, $5.2 \%$ pulmonary hypertension and $3.2 \%$ myocardiopathy. Regarding valvulopathies, $9,5 \%$ presented valvular dysfunction, $3.2 \%$ valvular thickening and $0.6 \%$ vegetation. The most frequently injured valve was the mitral $(9.1 \%)$, followed by the aortic $(2.8 \%)$. The majority of patients $(88.26 \%)$ were asymptomatic at the time of TTE. However, patients with pathological TTE had more dyspnea than those in the normal TTE group $(24.7 \%$ vs. $5.8 \%, p<0.001)$. Presenting a pathological TTE was associated with higher SLICC score $(p<0.001)$, greater number of admissions $(p<0.001)$ and mortality $(p=0.002)$. A higher SLEDAI was also associated with higher mortality $(p<0.001)$.

Conclusion: Cardiac involvement in SLE is not only related to damage accrual but can also be an early manifestation (beyond pericarditis), especially in active SLE. TTE assessment should be considered as a part of routine examination for SLE due to the high prevalence of heart disease even in asymptomatic patients.

\section{References:}

[1] Doria A, laccarino L, Sarzi-Puttini P, Atzeni F, Turriel M, Petri M. Cardiac involvement in systemic lupus erythematosus. Lupus. 2005;14(9):683-686.

[2] Chen J, Tang Y, Zhu M, Xu A. Heart involvement in systemic lupus erythematosus: a systemic review and meta-analysis. Clin Rheumatol. 2016; 35(10):2437-2448.

Disclosure of Interests: Jorge Álvarez Troncoso: None declared, Ángel Robles Marhuenda: None declared, Francesca Mitjavila Villero: None declared, Francisco José García Hernández: None declared, Adela Marín Ballvé: None declared, Antoni Castro Consultant of: Actelion pharmaceuticals, GSK, MSD., Gonzalo Salvador Cervelló: None declared, Eva Fonseca: None declared, Isabel Perales Fraile: None declared, Guillermo Ruiz-Iras torza: None declared DOI: 10.1136/annrheumdis-2020-eular.1633

\section{THU0257 ESTIMATED 10-YEARS CARDIOVASCULAR RISK IN SYSTEMIC LUPUS ERYTHEMATOSUS PATIENTS: PRELIMINARY RESULTS FROM THE "CARDIOVASCULAR OBESITY AND RHEUMATIC DISEASE (CORDIS)" STUDY GROUP OF THE ITALIAN SOCIETY OF RHEUMATOLOGY.}

F. Cacciapaglia ${ }^{1}$, A. Manfredi ${ }^{2}$, G. Erre ${ }^{3}$, E. Bartoloni Bocci ${ }^{4}$, G. Sakellariou ${ }^{5}$ O. Viapiana ${ }^{6}$, S. Colella ${ }^{1}$, A. Abbruzzese ${ }^{1}$, M. Fornaro ${ }^{1}$, G. Cafaro ${ }^{4}$, M. A. Fenu ${ }^{7}$, B. L. Palermo ${ }^{5}$, M. Dessì , A. Palermo ${ }^{2}$, A. Giollo ${ }^{8}$, E. Gremese ${ }^{9}$, F. R. Spinelli ${ }^{10}$ F. Atzeni ${ }^{11}, \mathrm{M}$. Piga ${ }^{7}$ on behalf of "Cardiovascular Obesity and Rheumatic DISease (CORDIS)" Study Group of the Italian Society of Rheumatology. ${ }^{1}$ Rheumatology Unit, Department of Emergency and Organs Transplantation - University and AOU Policlinico of Bari, Bari, Italy; ${ }^{2}$ Rheumatology Unit, University of Modena and Reggio Emilia, Azienda Ospedaliero-Universitaria Policlinico di Modena, Modena, Italy; ${ }^{3}$ UOC Reumatologia, Specialità Mediche - Azienda Ospedaliero Universitaria di Sassari, Sassari, Italy; ${ }^{4}$ Rheumatology Unit, Department of Medicine, University of Perugia., Perugia, Italy; ${ }^{5}$ Division of Rheumatology, University of Pavia, IRCCS Policlinico San Matteo Foundation, Pavia, Italy; ${ }^{6}$ Rheumatology Unit, University of Verona, Verona, Italy; ${ }^{7}$ Rheumatology Unit, Department of Medical Sciences - University and AOU Policlinico of Cagliari, Cagliari, Italy; ${ }^{8}$ Rheumatology Unit, University of Verona, 
Verona, Italy; ${ }^{9}$ Division of Rheumatology, Fondazione Policlinico Universitario A. Gemelli IRCCS, Università Cattolica del Sacro Cuore, Rome, Italy, Rome, Italy; ${ }^{10}$ Rheumatology Unit, Department of Internal Medicine and Medical Speciality, Sapienza University of Rome, Rome, Italy; ${ }^{11}$ Rheumatology Unit, University of Messina, Messina, Italy

Background: Systemic lupus erythematosus (SLE) patients are at high risk for CV events, and EULAR recommends assessing the 10-year CV-risk using the Systematic Coronary Risk Evaluation (SCORE) [1]. The QRISK3, another score to assess CV-risk in UK population, considers different factors among which also SLE. The Progetto Cuore score (PCS) is validated to estimate CV risk in Italian people and largely replicates the SCORE project [2].

Objectives: This cross-sectional study aimed to estimate CV-risk using SCORE, QRISK3 and, for the first time, PCS in a multicentric cohort of Italian SLE patients. Methods: During 2019 we evaluated 173 SLE patients (87.7\% female; age $40 \pm 16$ years; disease duration $138 \pm 105$ months), fulfilling the 1997 ACR classification criteria. Clinical and laboratory data were registered, and individual CV-risk was calculated using suitable algorithms for the SCORE, QRISK3 and PCS. Statistical analysis was performed using Graphpad Instat 8.0 (San Diego, CA-USA).

Results: In $13(7 \%)$ SLE patients a previous CV event was recorded. Hypertension was present in $60(37.5 \%)$ and diabetes in $27(16.9 \%)$ patients. Mean total cholesterol was $184 \pm 39 \mathrm{mg} / \mathrm{dL}$, HDLc $58 \pm 18 \mathrm{mg} / \mathrm{dL}$, LDLc $124 \pm 37 \mathrm{mg} / \mathrm{dL}$, triglycerides $105 \pm 63 \mathrm{mg} / \mathrm{dL}$; dyslipidaemia was reported in $58(36.2 \%)$ patients and $29(18.1 \%)$ were on statin. Mean BMI was $24.9 \pm 5.3 \mathrm{Kg} / \mathrm{sm}, 60(37.5 \%)$ and $23(14.3 \%)$ patients were overweight and obese, while 25 (15.6\%) patients were smokers. $87(54.3 \%)$ SLE patients had a SLEDAl $<4,91 \%$ of patients were taken $\mathrm{HCQ}$ and $65 \%$ were on prednisone (mean dose $5.4 \pm 5.9 \mathrm{mg} /$ day), but only $7.5 \%$ took $>7.5 \mathrm{mg} /$ day. The CV-risk of SLE patients according to SCORE, QRISK3 and PCS was $1.1 \pm 2.1 \%, 10.5 \pm 12.3 \%$ and $3.7 \pm 5.4 \%$, respectively. Stratifying patients at low, moderate or high $\mathrm{CV}$ risk according to the PCS and SCORE a double proportion of patients was at moderate ( $8 \%$ vs $3.9 \%$ ) or high $(1.9 \%$ vs $0.9 \%) \mathrm{CV}$ risk ( $p=0.03$ ). Finally, CV-risk according to QRISK3 was higher than $20 \%$ (high risk) in $32 / 160(20 \%)$ patients.

Conclusion: This multicentre study demonstrated that the mean estimated CV-risk in SLE patients is globally low using the SCORE, QRISK3 and PCS. The PCS seems to better intercept those patients at moderate/high risk, at least in Italian SLE patients, while QRISK3 predicts the highest CV risk. The lack of disease-specific CV-risk factors (such as autoantibodies profile or organ involvement) probably account for the underestimation of $\mathrm{CV}$ risk using the SCORE and PCS.

References:

[1] ARD 2019;78(6):736-745.

[2] ARD 2019;0:1-2. doi:10.1136/annrheumdis-2019-215715

Disclosure of Interests: Fabio Cacciapaglia Speakers bureau: BMS; Roche; Pfizer; Abbvie, Andreina Manfredi: None declared, Gianluca Erre: None declared, Elena Bartoloni Bocci: None declared, Garifallia Sakellariou Speakers bureau: Abbvie, Novartis, MSD, Ombretta Viapiana: None declared, Sergio Colella: None declared, Anna Abbruzzese: None declared, Marco Fornaro: None declared, Giacomo Cafaro: None declared, Maria Antonietta Fenu: None declared, Bianca Lucia Palermo: None declared, Martina Dessì: None declared, Adalgisa Palermo: None declared, Alessandro Giollo: None declared, Elisa Gremese Consultant of: AbbVie, Bristol-Myers Squibb, Celgene, Eli Lilly, Janssen, Merck Sharp \& Dohme, Novartis, Sanofi, UCB, Roche, Pfizer, Speakers bureau: AbbVie, Bristol-Myers Squibb, Celgene, Eli Lilly, Janssen, Merck Sharp \& Dohme, Novartis, Sanofi, UCB, Roche, Pfizer, Francesca Romana Spinelli Grant/research support from: Pfizer, Speakers bureau: Lilly, BMS, Celgene, Fabiola Atzeni: None declared, Matteo Piga: None declared

DOI: 10.1136/annrheumdis-2020-eular.6187

\section{THU0258 \\ SJOGREN'S SYNDROME WITH AND WITHOUT AND AUTOIMMUNE THYROIDITIS: IS THERE ANY DIFFERENCE?}

A. I. Celia ${ }^{1}$, S. Colafrancesco ${ }^{1}$, A. Gattamelata ${ }^{1}$, R. Izzo ${ }^{1}$, F. Giardina ${ }^{1}$, L. Mastromanno', R. Priori'. ' 'Sapienza University of Rome, Dipartimento di Scienze Cliniche, Internistiche, Anestesiologiche e Cardiovascolari, Rome, Italy

Background: Sjögren's syndrome (SS) is a systemic autoimmune disease mainly affecting exocrine glands and characterized by a progressive lymphocytic infiltration of salivary and lacrimal glands with consequent loss of function and development of sicca symptoms. Autoimmune thyroiditis (AT) is the most frequent autoimmune disease associated with SS and detectable in about $10-30 \%$ of cases ${ }^{1}$. Interestingly, patients with concomitant SS and AT seem to display a more attenuated phenotype compared to patients with solely SS. It is also noteworthy that up to $30 \%$ of patients with AT experience sicca symptoms without a clear diagnosis $\mathrm{SS}^{2}$. At the light of these evidences, it is unclear whether SS and AT represent two distinct nosological entities or different expressions of the same pathology.

Objectives: Aim of this study is to evaluate the prevalence of AT in a large monocentric cohort of patients with SS and to define its clinical and laboratory characteristics compared to isolated SS.

Methods: Consecutive patients with SS (AECG criteria) referring to our "Sjögren Clinic" (Sapienza University of Rome) were enrolled and divided in two groups: SS with AT (group 1) and SS without AT (group 2). Group 1 was further divided in two subgroups depending on the presence (1a) or absence (1b) of anti Ro/SSA antibodies. The following clinical and laboratory data were retrospectively collected for all patients: concomitant celiac disease, arthralgia, lung involvement, purpura, lymphoma, presence of ANA, anti-Ro/SSA, anti-La/ SSB, rheumatoid factor, cryoglobulins, leukopenia and hypergammaglobulinaemia. These characteristics were compared between the following groups: group 1 , group 2, group $1 \mathrm{a}$ and $1 \mathrm{~b}$. For statistic Chi Square and Fisher's test analysis were performed.

Results: Six-hundred and three SS patients were enrolled (group $1 \mathrm{n=135}$; group $2 \mathrm{n}=381$; group 1a $\mathrm{n}=96$; group $1 \mathrm{~b} \mathrm{n}=39$ ). The prevalence of AT was 135/603 (22.3\%). When comparing SS patients with or without AT (group 1 vs group 2) the frequency of rheumatoid factor was significantly higher in group 2 compared to group $1(p=0.006)$. No case of lymphoma was recorded in group 1 while 14 cases of lymphoma were ascertained in group $2(p=0,08)$. Conversely, celiac disease was higher in group 1 compared to group $2(p=0.01)$. No other differences between these groups were identified. Stratifying SS patients with AT according to the presence (group 1a) or not (group 1b) of anti Ro/SSA antibodies, ANA rheumatoid factor and hypergammaglobulinemia were significantly more positive in group 1 a compared to group $1 \mathrm{~b}(\mathrm{p}=0.0002, \mathrm{p}=0.002, \mathrm{p}=0.02$, respectively); no clinical differences were identified.

Conclusion: In this study, we confirm the presence of a less aggressive disease in patients with SS and AT compared to solely SS. The higher prevalence of rheumatoid factor and lymphoma occurrence in SS without AT, strictly suggest a more severe phenotype in this subset. Although is known that in SS patients with anti Ro/SSA+ antibodies and RF there is a more aggressive disease, in SS with AT the presence or absence of such autoantibodies do not seems to associate with any difference in clinical severity. Follow up studies are presently being carried out in order to provide conformation of a less sever phenotype and a better disease outcome in patients with associated SS and AT.

\section{References:}

[1] Jara, L.J., Navarro, C., Brito-Zerón, M.P. et al. Thyroid disease in Sjögren's syndrome. Clin Rheumatol 26, 1601-1606 (2007)

[2] Vera D. Milic, Goran Radunovic, Ivan Boricic, Sanja Ognjanovic, Radmila Petrovic, Marija Radak-Perovic, Nada Vujasinovic-Stupar, Nemanja Damjanov, High prevalence of autoimmune thyroid disease in subjects with sicca symptoms without Sjögren's syndrome, Rheumatology, Volume 52, Issue 4 April 2013, Pages 754-755.

Disclosure of Interests: None declared

DOI: 10.1136/annrheumdis-2020-eular.6059

\section{THU0259 \\ LYMPHOMAS IN ANTICENTROMERE ANTIBODY POSITIVE PRIMARY SJOGREN'S SYNDROME}

B. Chaltcev ${ }^{1}$, V. Vasiliev ${ }^{2}$, S. Palshina ${ }^{1}$, J. Khvan ${ }^{1}$, E. Sokol ${ }^{1}$, A. Torgashina ${ }^{1}$

${ }^{1}$ V.A. Nasonova Research Institute of Rheumatology, Moscow, Russian

Federation; ${ }^{2}$ MEDSI, Moscow, Russian Federation

Background: Patients with primary Sjogren's syndrome (pSS) are at high risk of lymphoma. Signs of lymphomas in ACA+pSS were not widely reported, the descriptions were single [1, 2]. According to pSS treatment recommendations [3, $4,5,6]$, glandular forms require only symptomatic treatment, in the absence of systemic manifestations systemic therapy is not required.

Objectives: to evaluate the incidence of lymphomas in ACA+pSS; to compare clinical and laboratory manifestations in 2 groups of ACA+pSS: with and without lymphomas.

Methods: we examined 119 ACA+pSS patients. We evaluated both glandular and systemic manifestations. We diagnosed lymphomas based on study of biopsy specimens of affected organs.

Results: MALT-lymphomas diagnosed in 19 (16\%) ACA+pSS patients. Persistent enlargement of parotid salivary glands, decreased C4-complement, decreased CD19+cells in peripheral blood, cryoglobulinemic vasculitis, lymphoid infiltration of minor salivary glands (MSG) more than 200 cells in focus, severe xerostomia and xerophthalmia more often detected in patients with ACA+pSS+lymphomas. RF and antiRo were found in only $25 \%$ of patients with lymphomas and its 